\title{
COVID-19 infection during pregnancy: fetus as a patient deserves more attention
}

https://doi.org/10.1515/jpm-2020-0181

Received April 25, 2020; accepted May 3, 2020; previously published online May 13, 2020

Abstract: The novel coronavirus disease 2019 (COVID-19) pandemic is causing concern also for the management and outcome of COVID-19-positive pregnant women and their offspring, as reported cases are rare. Current evidence suggests the association of COVID-19 infection in pregnancy with both severe maternal morbidity requiring intensive care and perinatal complications (preterm birth with consequent neonatal morbidity and even perinatal death). Most of the reported cases focused specifically on the maternal outcomes and possible vertical transmission, but less attention has been paid to fetus as a patient in such pregnancies. The use of antenatal steroids and fetal neuroprotection with magnesium sulfate is clearly underreported. Several recently issued guidelines suggest lowering the upper gestational age for antenatal steroid administration and also advocate extreme caution or even restraining from the use of magnesium sulfate. Also, the rate of cesarean deliveries among COVID-19 women is unacceptably high. Here we provide arguments for NOT changing the existing guidelines and caution against cesarean delivery that was the prevalent delivery mode in the reported cases and case series.

Keywords: antenatal steroids; COVID-19; delivery mode; fetal neuroprotection; pregnancy.

\section{Background}

The novel coronavirus disease 2019 (COVID-19) pandemic is causing concern and rapid adjustment within the field of obstetrics and fetal medicine. Current evidence suggests the association of COVID-19 infection in pregnancy with both severe maternal morbidity requiring intensive

\footnotetext{
*Corresponding author: Prof. Vedran Stefanovic, Department of Obstetrics and Gynecology, Fetomaternal Medical Center, Helsinki University Hospital and University of Helsinki, Haartmaninkatu 2, 00290 Helsinki, Finland, Tel.: +358 50 4271230,

E-mail:vedran.stefanovic@hus.fi
}

care and perinatal complications (preterm birth with consequent neonatal morbidity and even perinatal death). While most of the reported cases focused specifically on the maternal outcomes and possible vertical transmission, less attention has been paid to fetus as a patient in such pregnancies. For example, majority of publications are systematically lacking data on antenatal steroid use and fetal neuroprotection which are the cornerstones of the antenatal interventions for the improvement of outcome in premature delivery, frequently associated with COVID-19 infection. Also, changes to existing guidelines concerning antenatal interventions caused by COVID-19 pandemic are more rule than exception with scarce or no evidence-based approach for such adjustments.

\section{Antenatal steroids}

The use of antenatal steroids for the fetal lung maturation is a mainstay of therapy for women with anticipated preterm birth. The concern about the impact of antenatal steroid use on the outcome of critically ill patients with COVID-19 infection raised after several studies demonstrated that outcomes are worse for patients with COVID-19 infection with corticosteroid use [1, 2]. However, these studies were unable to control for underlying medical comorbidities, ventilation, or intensive care unit (ICU) status and no pregnant women were included. It is also unclear from the published results whether the steroids were given because the baseline condition of the critically ill patients was worse or if the steroid treatment was the cause of worse outcomes. Also, the dosage of steroids in an ICU setting is usually different than for obstetric indications regarding acceleration of intrauterine fetal lung maturation. For example, the typical obstetrical dosing of betamethasone in methylprednisolone equivalents is $60 \mathrm{mg}$ and is similar to those reported. However, the duration of reported treatment was long (4-11 days of treatment). Therefore, the typical antenatal steroid treatment used in pregnant women $(24 \mathrm{mg}$ of betamethasone in two divided doses $24 \mathrm{~h}$ apart intramuscularly) uses roughly one-fourth to one-tenth of the dosage used in these publications. This 
issue has been clearly discussed in the recent publication by McIntosh [3]. Translation of these results into the obstetrics, making assumptions and unjustified adjustments of the guidelines and lowering the gestational age for the administration of antenatal steroids may be potentially harmful.

It has to be acknowledged that the number of mothers needed to treat with corticosteroids to prevent one neonatal death is six at 23-24 weeks but can increase to 798 women at 34 weeks [4]. However, $8 \%$ of all deliveries occur in the late preterm period. Thus, the potential public health and economic effects of decreasing the rate of complications associated with late prematurity by the administration of antenatal steroids are considerable. A randomized trial demonstrated that administration of betamethasone to women at risk for late preterm delivery significantly reduced the rate of neonatal respiratory complications [5].

\section{Delivery mode}

The majority of deliveries in published COVID-19 pregnancies occur by elective or emergency cesarean section (CS), although the greatest proportion of such pregnancies are asymptomatic or with mild symptoms [6]. Apart from the substantial maternal morbidity associated with $\mathrm{CS}$, it may be harmful for the neonate, as well. A recently published meta-analysis that included 26 studies showed that both elective and emergency CS were associated with an increased risk of neonatal respiratory distress syndrome [7]. In addition, the associations of CS and elective CS with the risk of neonatal respiratory distress syndrome (RDS) remained significant in subgroup analysis by continent, study design, number of fetuses, and adjustment status of gestational age, birthweight, and maternal age. In addition, this meta-analysis demonstrated that elective CS significantly increased the risk of neonatal RDS also for full-term newborns. Similar results were demonstrated in the large retrospective study [8].

The true reason for the overrepresentation of cesarean deliveries in COVID-19-positive pregnant women may not be identified on the basis of current reports. As the details of such pregnancies are very scarce, we speculate that deterioration of the patient's condition may happen abruptly with consequent oxygenation problems leading to the signs of fetal distress and in such a situation labor induction is not possible. The other reason may be more "logistic" in nature. Very often COVID-19 pregnant women are treated outside of the delivery wards and vaginal labor may be challenging in such situations and environment.

\section{Magnesium sulfate for fetal neuroprotection}

It has been undisputably demonstrated that antenatal magnesium sulfate given prior to preterm birth for fetal neuroprotection prevents cerebral palsy (CP) and reduces the combined risk of fetal/infant death or CP [9]. This policy has been widely implemented, especially in pregnancies with imminent preterm birth before 32 gestational weeks. Recently, several publications dealing with antenatal interventions in COVID-19-positive women expressed concern about the possible detrimental impact of magnesium sulfate on the respiratory depression in pregnant women with severe COVID-19 disease. Appropriate administration of antenatal magnesium sulfate was not shown to be associated with serious maternal adverse effects [10]. Interestingly, magnesium has also a dilatory effect on all smooth muscles and serves as a bronchodilator showing even beneficial effects on the pulmonary function in patients with severe asthma and decreasing production of reactive oxygen species (ROS), which are elevated in patients with acute respiratory distress syndrome [11, 12]. The only determinant of magnesium serum concentration is renal clearance, so there is no reason NOT to administer fetal neuroprotection in pregnant women with COVID-19 infection and normal renal function. Monitoring of diuresis ( $\geq 30 \mathrm{~mL} / \mathrm{h}$ ) and/or basic laboratory evaluation of the renal function should be appropriate measures to avoid magnesium toxicity without the need for magnesium concentration measurement. Magnesium sulfate infusion is easily prepared and monitored. It should be reminded that fetal neuroprotective benefit may be achieved even by administration of only the loading dose as either $4 \mathrm{~g}$ of magnesium sulfate intravenous bolus or $6 \mathrm{~g}$ intravenous infusion over 20-30 min if clinical situation does not allow maintenance dose administration.

\section{Conclusion}

Based on the current evidence, we suggest that COVID-19 pandemic should NOT trigger the changes in the existing widely accepted guidelines on antenatal steroids and fetal neuroprotection with magnesium sulfate. Also, in the absence of other "common" obstetrical indications, CS as 
a delivery mode for pregnant women with COVID-19 infection should be reserved only for severe forms of disease with abrupt deterioration. Currently, there is only one publication that explicitly made a statement: "Before any preterm delivery, antenatal corticosteroids and magnesium sulfate for neuroprotection should be given to any mother with a viable fetus" [13]. We hope that authoritative societies for gynecology, obstetrics, and fetal and perinatal medicine should release promptly clear statements on these issues to avoid confusion and potential harms to mothers, fetuses, and neonates.

Research funding: None declared.

Author contributions: The author has accepted responsibility for the entire content of this manuscript and approved its submission.

Competing interests: Author states no conflict of interest. Informed consent: Informed consent was obtained from all individuals included in this study.

\section{References}

1. Guan WJ, Ni ZY, Hu Y, Liang WH, Ou CQ, He JX, et al.Clinical characteristics of coronavirus disease 2019 in China. N Engl J Med 2020;382:1708-20.

2. Zhou F, Yu T, Du R, Fan G, Liu Y, Liu Z, et al. Clinical course and risk factors for mortality of adult inpatients with COVID-19 in Wuhan, China: a retrospective cohort study. Lancet 2020;395:1054-62.

3. McIntosh JJ. Corticosteroid Guidance for Pregnancy during COVID19 Pandemic. Am J Perinatol 2020. doi: 10.1055/s-0040-1709684.
4. Travers CP, Clark RH, Spitzer AR, Das A, Garite TJ, Carlo WA. Exposure to any antenatal corticosteroids and outcomes in preterm infants by gestational age: prospective cohort study. $\mathrm{Br}$ Med J 2017;356:j1039.

5. Gyamfi-Bannerman C, Thom EA, Blackwell SC, Tita AT, Reddy UM, Saade GR, et al. Antenatal betamethasone for women at risk for late preterm delivery. N Engl J Med 2016;374:1311-20.

6. Zaigham M, Andersson 0 . Maternal and perinatal outcomes with COVID-19: a systematic review of 108 pregnancies. Acta Obstet Gynecol Scand 2020. doi: 10.1111/aogs.13867.

7. Li Y, Zhang C, Zhang D. Cesarean section and the risk of neonatal respiratory distress syndrome: a meta-analysis. Arch Gynecol Obstet 2019;300:503-17.

8. Baumert M, Fiala M, Walencka Z, Paprotny M, Sypniewska K. Cesarean delivery and respiratory distress in late preterm and term infants. Cent Eur J Med 2012;7:230-4.

9. Crowther CA, Middleton PF, Voysey M, Askie L, Duley L, Pryde $P G$, et al. Assessing the neuroprotective benefits for babies of antenatal magnesium sulphate: an individual participant data meta-analysis. PLoS Med 2017;14:e1002398.

10. Bain ES, Middleton PF, Crowther CA. Maternal adverse effects of different antenatal magnesium sulphate regimens for improving maternal and infant outcomes: a systematic review. BMC Pregnancy Childbirth 2013;13:195.

11. Shan Z, Rong Y, Yang W, Wang D, Yao P, Xie J, et al. Intravenous and nebulized magnesium sulfate for treating acute asthma in adults and children: a systematic review and meta-analysis. Respir Med 2013;107:321-30.

12. Schmidt R, Luboeinski T, Markart P, Ruppert C, Daum C, Grimminger $\mathrm{F}$, et al. Alveolar antioxidant status in patients with acute respiratory distress syndrome. Eur Respir J 2004;24:994-9.

13. Chen D, Yang H, Cao Y, Cheng W, Duan T, Fan C, et al. Expert consensus for managing pregnant women and neonates born to mothers with suspected or confirmed novel coronavirus (COVID-19) infection. Int J Gynecol Obstet 2020;149:130-6. 